\title{
Optimal fatigue analysis of structures during complex loadings
}

\author{
Habib Karaouni ${ }^{\text {a,* }}$ and Joseph Zarka \\ Laboratoire de Mécanique des Solides, École polytechnique, 91128 Palaiseau Cedex, France
}

Received 23 April 2016 / Accepted 2 September 2016

\begin{abstract}
A new framework for high cycle fatigue analysis of metallic structures under complex multi-parameter loadings was here developed. This allows to reduce the analysis on a 2-D window with a characterized one-parameter cyclic loading thanks to an equivalence rule relative to damage between any two loadings. The simplified inelastic analysis introduced by J. Zarka [J. Zarka et al. 1990. A new approach in inelastic analysis of structures. CADLM] was used to find the limit state of the structure. A new design rules for fatigue analysis by utilizing automatic learning systems was successfully performed. A database was built by coupling numerical simulations and experimental results on several welded specimens which are considered as a general structure in the proposed approach. This could be possible by the introduction of an intelligent description of a general fatigue case based on the actual theories and models. A software, FATPRO [M.I. Systems, FatPro, available at http://www.mzintsys.com/our_products_ fatpro.html], based on this work has been developed at MZ Intelligent Systems.
\end{abstract}

Key words: Fatigue, Machine Learning, Optimal Design, Inelastic analysis, Multi-axial loadings.

\section{Introduction}

Many mechanical and structural components are subjected to complex loadings in service. Many accidents were consequences of inaccurate estimation of stresses and fatigue life. Despite many years of work and research on the subject, fatigue life estimation is still very important to be examined.

During classical analysis, a linear or non-linear finite element simulation is usually performed, and then eventually with some special methods to extract and to count the cycles from the random stress path (very often, the rainflow method), it is needed to cumulate "damage" based on some S-N diagrams and the linear Miner-Palmgreen rule or some more elaborated nonlinear rules. The "damage" factor (scalar or tensor) is still the object of many researches; it is impossible to measure it during the whole loading path, even if, before failure; some slight changes on the elastic properties may be experimentally detected. Moreover, the representations of the loading and the counting methods are purely based on mathematical aspects and ignore the particular mechanical behavior of the present materials in the structure.

The objective here is at first to describe an approach which has a real physical meaning and which is very easy to perform. The principal idea is to find an equivalent simple loading suitable to represent the real random loading in terms of damage produced at the level of the material then at the level of the structure.

We introduced a global representation of any loading at the scale of the material to give the equivalence rule between any two loadings at the scale of the material and to allow to represent any random loading by an equivalent periodic loading. We chose as a "measure" of any one-parameter random loading, the cumulated plastic strain (or equivalently the dissipated energy) at a NEW very local level (similarly to what it is done during a seismic analysis). Two loadings are equivalent if they induce the same cumulated plastic strain (or the same dissipated energy).

The other objectives refer to that during fatigue analysis:

(i) we have to take into account the uncertainties on geometry, material properties, loadings, etc.

(ii) we have no knowledge of the real initial state, etc.

(iii) and also, we cannot control the errors during numerical simulations or measurements.

To overcome these difficulties a NEW FRAMEWORK FOR FATIGUE ANALYSIS AND RELIABILITY OF STRUCTURES has been built which allows the analysis to be reduced to the analysis on a 2-D window with a characterized one-parameter cyclic loading. It is given ONE DESCRIPTION for the smooth

\footnotetext{
a Present address: Safran Tech, Rue des Jeunes Bois, 78772 Magny-les-Hameaux, France.

*e-mail: habib.karaouni@safrangroup.com 
specimen and any general structure with any loading conditions; simplified analysis of inelastic structures [1] and simple rules for damage and accumulation are taken BUT the results are COUPLED WITH RESULTS FROM RETURNS ON REAL STRUCTURES.

All these procedures and framework were introduced in a software, FatPro [2], that is developed and distributed by MZ Intelligent Systems.

\section{Review on the intelligent optimal design of materials and structures}

All details may be found in the book [3].

\subsection{Automatic learning expert systems}

The engineers have to face very important problems in the design, the test, the survey and the maintenance of their structures. Moreover, the available data may be not statistically representative (i.e. are in limited number), and may be fuzzy, qualitative and/or missing in part. Other methods need to be used!

An AUTOMATIC LEARNING EXPERT SYSTEMS Generator is able to automatically extract the rules from the raw examples base given by the expert. Their main problem is to provide a good description of such an examples base. (By analogy, we can say that the data base is the program, the automatic learning tool is the compiler and the execution gives the knowledge).

Basically such an automatic learning system includes five main functions: PREPARE data, LEARN rules, TEST and visualize INCLEAR the extracted rules, and used them to CONCLUDE new cases.

In all problems, it is necessary to consider one conclusion which may be a class or any continuous real number. Moreover, often, several conclusions may be considered together. The rules have to be automatically generated for each one of them.

Then an optional but fundamental sixth function, OPTIMIZE, based often on genetic algorithms and other special optimization techniques, may be used to solve the inverse problem i.e. when some conclusions and some descriptors have to belong to some given sets (or constraints), what are the possible solutions and in some particular cases what is the best solution if an objective function is given (cost, weight.)?

\subsection{General principle of the approach}

We have defined a new approach [3] where it is needed:

1. To build a DATABASE of examples i.e. to obtain some experimental, real or simulated results where the EXPERTS indicate all variables or descriptors this may take a part. This is, at first, done with some PRIMITIVE descriptors $x$, which are usually in a limited number and which are often in a different number and type for each example. Then, the data are transformed with the introduction of some INTELLIGENT descriptors $X X$, with the actual whole knowledge thanks to (but often insufficient) beautiful theories and models. These descriptors may be number, Boolean, strings, names of files which give access to data bases, or treatments of curves, signals and images. But for all examples, their number and their type are always the same, which is the only one way to allow the fusion of data. The results or conclusions may be classes (good, not good...) or numbers. Usually, it is hoped to be possible to get $\sim 50-500$ examples in the data base with 10-1000 descriptors, 1-20 conclusions for each case. This is the MOST IMPORTANT (and DIFFICULT) TASK.

2. To generate the RULES with any Automatic Learning Tool. Each conclusion is explained as function or set of rules of some among the input intelligent descriptors with a known reliability or accuracy. If this reliability is too low, that means that there is not enough data or we have bad or missing intelligent descriptors.

3. To optimize at two levels (Inverse Problems)

- Considering the intelligent descriptors as independent; it is possible to get the OPTIMAL SOLUTION satisfying the special required properties and allowing the DISCOVERY OF NEW MECHANISMS,

- Considering the intelligent descriptors linked to primitive descriptors for a special family; it is possible to obtain the optimal solution that is technologically possible.

So, not only a Practical Optimal Solution is obtained but also the Experts may learn the missing parts, may build models or theories based only on the retained intelligent descriptors and guided by the shapes of the rules or relationships.

In order to follow our "intelligent" approach for the fatigue analysis, we need to create a data base of examples/ problems. Each case/problem is at first defined by its primitive description. There is no way, with such a description, to use the results obtained from one case into another case/problem. The intelligent description has then to be performed.

Since, we cannot rely to the numerical (or experimental) results for the classical LOCAL dimensioning criteria, we proposed to perform only the cheapest elastic analysis or our simplified analysis of inelastic structures [1] and to use only the simplest rules for damage criteria and cumulation of damage for materials but we shall couple them with the results from returns on real structures.

\section{Fundamental data on materials}

On a smooth tensile specimen, it is easy to perform the following characterizations:

At first, the cyclic curves at $\sim \pm 1 \%$ of deformation are obtained (this is the best choice since the first tensile curve is a function of the initial state of the material, but it implies 
that the material is cyclically stable) eventually for different temperatures. Usually, these curves are represented by:

$$
E=E^{\mathrm{e}}+E^{\mathrm{p}}=\frac{\Sigma}{Y_{\mathrm{G}}}+\left(\frac{\Sigma}{K^{\prime}}\right)^{1 / n^{\prime}}
$$

where $K^{\prime}$ and $n^{\prime}$ are constants.

Then, the Wöhler curves are constructed during cyclic uniaxial loading, associated to various mean radial stresses and probabilities of failure. As proposed by the experts, they are represented by the stress-life equation:

$$
\Sigma_{\mathrm{a}}=\left(\sigma_{\mathrm{f}}^{\prime}-\Sigma_{\mathrm{m}}\right)\left(2 N_{\mathrm{f}}\right)^{b}
$$

$\Sigma_{\mathrm{a}}$ is the stress amplitude, and $\Sigma_{\mathrm{m}}$ is the mean stress and $\Sigma_{\max }=\Sigma_{\mathrm{a}}+\Sigma_{\mathrm{m}}$ is the maximum or peak stress.

Or, more practically, as in the Morrow's representation, Wöhler curves are represented by the strain-life equation:

$$
\frac{\Delta E}{2}=\frac{\Delta E^{\mathrm{e}}}{2}+\frac{\Delta E^{\mathrm{p}}}{2}=\frac{\sigma_{\mathrm{f}}^{\prime}}{Y_{\mathrm{G}}}\left(2 N_{\mathrm{f}}\right)^{b}+\varepsilon^{\prime}\left(2 N_{\mathrm{f}}\right)^{c}
$$

In this equation, $\Delta E, \Delta E^{\mathrm{e}}, \Delta E^{\mathrm{p}}$ are the strain, elastic strain and plastic strain ranges respectively, $2 N_{\mathrm{f}}$ is the number of cycles to failure and with the constitutive coefficients for the material, $\sigma_{\mathrm{f}}^{\prime}$ is the fatigue strength coefficient, $Y_{\mathrm{G}}$ is the Young modulus, $\varepsilon_{\mathrm{f}}^{\prime}$ is the fatigue ductility coefficient, $c$ is the fatigue ductility exponent, and $b$ is the fatigue strength exponent.

Then, the endurance diagram is constructed. A modified Goodman's diagram is usually employed for a given number of cycles, $N$, in the plane $\left(\Sigma_{\mathrm{m}} ; \Sigma_{\mathrm{a}}\right)$ a bounded domain is defined. Next, the creep time to rupture is extracted.

During complex uniaxial loading, different deterministic mathematical representations are based on peak-valley or range-mean matrix type to define groups of constant amplitude cycles, each with a fixed load amplitude and mean value. Such as cumulative exceeding curves (with the same reduction or counting of cycles) or sequential variable amplitude histories (usually the rainflow method [4] of counting the cycles is preferred). During each of these groups of constant amplitude cycles some "damage" is induced. Various rules are proposed to cumulate the "damage". Linear ones by Palmgreen-Miner:

$$
D_{i}=\frac{N_{i}}{N_{r_{i}}}
$$

and failure when:

$$
\sum_{i} D_{i}=1 \Longleftrightarrow \sum_{i} \frac{N_{i}}{N_{r_{i}}}=1
$$

where $N_{i}$ is the number of cycles realized during the cyclic loading $i$ for which the number of cycles to rupture is $N_{r_{i}}$.

Or nonlinear ones such as those by Lemaitre and Chaboche [5].

The probabilistic representation (with the power spectra density to measure the load amplitude intensity in the frequency range) is often employed during random (stationary gaussian) uniaxial loadings; it may be only combined with the linear cumulative rule for damage. Several experts are thinking that non gaussian uniaxial loadings are more realistic and need special treatments [6].

During cyclic multiaxial loading (several loading parameters inducing out-of-phase stresses), the critical plane approach seems to be reasonable. For non-cyclic multiaxial loadings, we shall only keep the simplest endurance criteria such that proposed by Dang Van [7] or Kakuno-Kawada [8].

Generally, it has the form:

$$
C_{\mathrm{a}}+\alpha P_{\mathrm{a}}+\beta P_{\text {mean }}<b
$$

where $C_{\mathrm{a}}$ is the deviatoric stress amplitude, $P_{\mathrm{a}}$ the amplitude and $P_{\text {mean }}$ the mean value of the pressure (first invariant of the stress tensor). $\alpha, \beta$ and $b$ are some phenomenological constants of the material.

The Dang Van's criterion is for example, written locally:

$$
F^{\mathrm{DV}}=\max _{t}\left[\max _{\vec{n}}\left[\frac{\tau(\vec{n}, t)+a_{\mathrm{DV}} p(t)}{b_{\mathrm{DV}}}\right]\right]
$$

where $\tau(\vec{n}, t)$ is the local shear stress on the slip plane with normal $\vec{n}, p(t)$ is the local pressure and $a_{\mathrm{DV}} b_{\mathrm{DV}}$ are constants identified from endurance limits during alternate flexion and torsion tests.

These local quantities may be expressed from the global ones by making some simplifications:

$$
F^{\mathrm{DV}}=\max _{t}\left[\frac{\tau(t)+a_{\mathrm{DV}} p(t)}{b_{\mathrm{DV}}}\right]
$$

where $\tau(t)$ is the alternate maximal shear stress which is computed from the alternate part of the macroscopic deviatoric stress and $P(t)$ is the global pressure.

But there are still many open problems:

1. What is the validity of these Fatigue/Endurance criteria?

2. What is the real physical meaning of the Damage parameter(s)?

3. How to extract or represent of the random loading?

4. How to define the cumulative rules for Damage?

Indeed, it seems for us, that there is NO DIFFERENCE BETWEEN "GOD" with several religions and churches and DAMAGE with several models/theories and schools!

That is why, we shall take all of them.

\section{Intelligent representation of any loading}

The most difficult part is to describe the loading. The main idea of the approach is to find an Equivalence rule between TWO loadings relative to "damage" which may be used as a "quantification" or norm of any loading relative to one particular structure made of particular materials as introduced by Karaouni [8]. This rule needs to give a physical meaning of fatigue analysis during any loading and must allow for construction of simple and practical tools for fatigue analysis or accelerated fatigue tests. This representation has to be considered at the level of the material and then at the level of the structure.

The material has a macroscopic behavior with its macroscopic elastic limit and work-hardening modulus with which direct or incremental analysis of the structure are 
Loading 1

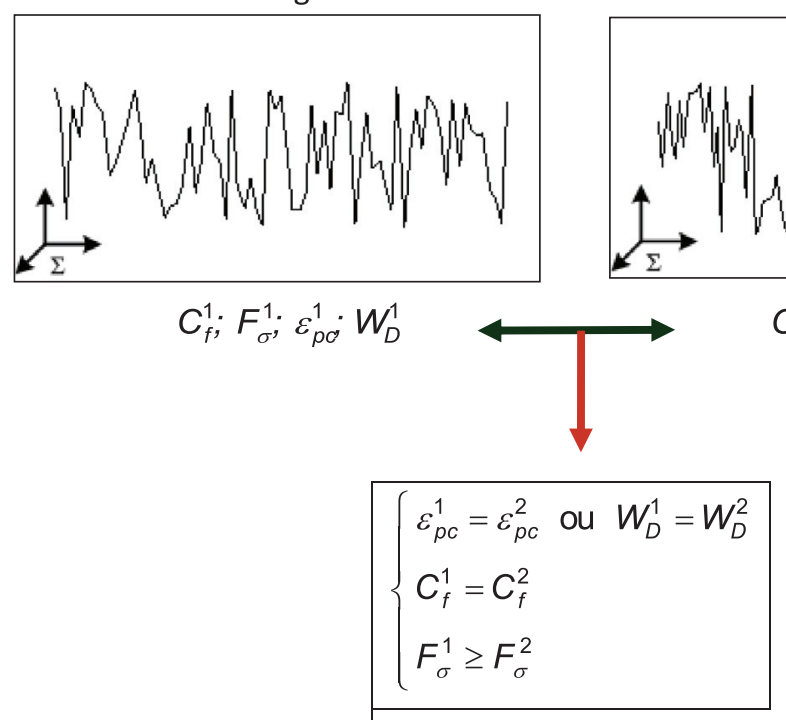

Loading 2

$C_{f}^{2} ; F_{\sigma}^{2} ; \varepsilon_{p \sigma}^{2} ; W_{D}^{2}$

Figure 1. Equivalence rule between two one-parameter random loadings.

performed. As only high cycle fatigue is considered in this work, elastic shakedown of the structure is reached after some time.

But the material has a microscopic local behavior with its microscopic elastic or endurance limit and local workhardening modulus. Although the global elastic shakedown, there is a local plastic shakedown.

\subsection{Level of the material}

\subsubsection{One-parameter random loadings}

Any one dimensional loading has its Center $C_{\mathrm{f}}=$ $\left(\Sigma_{\max }+\Sigma_{\min }\right) / 2$, and its fluctuation $F_{\sigma}=\left(\Sigma_{\max }-\Sigma_{\min }\right) / 2$.

The local Cumulated plastic strain $\varepsilon_{\mathrm{pc}}$ or the local dissipated energy $W_{\mathrm{D}}$ during the real random loading is computed on the model representing the material such as, for example, the Kinematic Hardening behavior.

$$
\begin{gathered}
\varepsilon_{\mathrm{pc}}=\int_{0}^{t}\left\|\underline{\dot{\varepsilon}}_{\underline{p}}(u)\right\| \mathrm{d} u \\
W_{\mathrm{D}}(t)=\int_{0}^{t} \underline{\underline{\Sigma}}(u): \underline{\underline{\dot{\varepsilon}}}_{p}(u) \mathrm{d} u
\end{gathered}
$$

Two loadings are equivalent if they induce the same cumulative plastic strain or the same dissipated energy, they have the same center and almost the same fluctuation (Figure 1).

A particular cyclic equivalent loading can be later defined and will be represented by the triplet $\left(C_{\mathrm{f}} ; F_{\sigma}^{2}=\Delta \Sigma ; N\right)$, where $N$ being obtained by mean of the equality between the accumulated plastic deformations or the local dissipated energies.

\subsubsection{Multi-parameter random stress path}

Taking the case of loading with $M$ parameters, the same procedure will be used. In the space of loading parameters, the bounding surface now becomes an hyper-sphere defined by its center $C_{\mathrm{f}}^{\mathrm{va}}$ and its radius $F_{\sigma}^{\mathrm{va}}$.

The equivalent radial cyclic loading must meet the following conditions:

- same centre $C_{\mathrm{f}}^{\mathrm{eq}}=C_{\mathrm{f}}^{\mathrm{va}}$,

- about the same fluctuation $F_{\sigma}^{\mathrm{eq}}=\beta F_{\sigma}^{\mathrm{va}}$, where $\beta>1$ and so that $F_{\sigma}^{\mathrm{eq}}=2 \sigma_{y}$ (to insure the global adaptation),

- same cumulative plastic deformation.

$\sigma_{y}$ being the global elastic limit.

The equivalent radial loading, noticed in one direction $\theta$ will be written $\left(C_{\mathrm{f}}^{\mathrm{eq}} ; F_{\sigma}^{\mathrm{eq}} ; N\right)_{\theta}$ and must satisfy the following properties:

$$
\left\{\begin{array}{c}
\left(C_{\mathrm{f}}^{\mathrm{eq}} ; F_{\sigma}^{\mathrm{eq}} ; N\right)_{\theta}=\left(C_{\mathrm{f}}^{\mathrm{va}} ; \beta F_{\sigma}^{\mathrm{va}} ; N\right)_{\theta} \\
1 \leq \beta \leq \frac{2 \sigma_{y}}{F_{\sigma}^{\mathrm{va}}} \\
N \text { such as } \varepsilon \frac{\mathrm{eq}}{\mathrm{pc}}=\varepsilon \frac{\mathrm{va}}{\mathrm{pc}} \\
\theta: \text { direction of the local loading }
\end{array}\right.
$$

The number of equivalent cycles $\left(N_{\theta}\right)$ of the equivalent loading depends on the direction $\theta$. This loading could be used for example in accelerated traction-compression tests or alternative torsion tests (Figure 2).

As we keep the fluctuation always the same, the range of microscopic plastic strains will be the same (to keep the same unknown damage mechanisms), as it may be seen in the figures: the response to any stress path is bounded by the "circular" stress path (Figure 3).

Intelligent description of one general fatigue case.

It is needed to transfer the primitive, passive descriptions of the geometry of structure, of its various materials and of one block of its random loading parameters to an intelligent, active description such that the experimental results on one structure may be used to any other new one! 


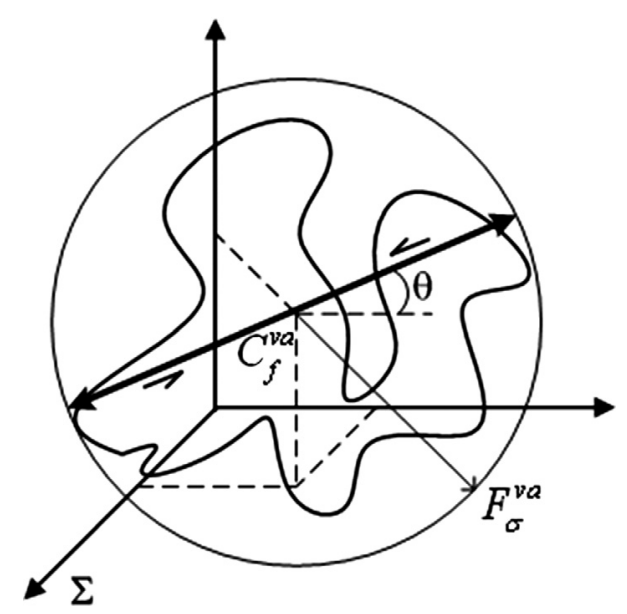

Figure 2. Centre and fluctuation for a multi-parameter random loading.

Here, in any point of the structure, the Output Primitive descriptors or conclusions are:

(i) failure or no failure and

(ii) when there is failure, the number of blocks of the loading.

A multi-scale elastic analysis is considered, for example for a naval structure (Figure 4):

(i) at the scale of the hull with rather crude mesh; elastic analysis is performed due to the various loadings,

(ii) then at the level of a substructure 3-D with a refined mesh (with the initial loading and the real loading, elastic, elastoplastic or simplified inelastic analysis are performed); the equivalent radial cyclic loading is characterized, and

(iii) then at the scale of a 2-D detail (one slice by a plane of the substructure) with a much refined mesh; a mobile window allows to focus at the different hot zones. The materials: base material, weld, and HAZ, are described from the material property database (smooth specimen data).

The moving window is similar to a filter. Its size is such that the quantities which are needed become rather insensitive to the errors associated to the mesh size and distribution. These quantities are related to the description of the stress and the gradient of this stress field. Among them, there are:

- The Maximum, Minimum and Average value of any field, such as $I_{1}$ and $J_{2}$, the Dang Van criterion, etc.;

- Inside the window, volumes where any fatigue criterion is violated by a factor of $50 \%, 80 \%$ or $100 \%$.

\section{Generation of the design rules to fatigue}

\subsection{Available experimental data}

All the experimental data used in the study are coming from the tests which were performed at the Illinois
University [9]. These tests were done in order to compare various design methods and to analyze the influence of some loading parameters and of the geometry on marine welded structures.

Several structures, indeed several characteristic details were tested. They were selected among the classical ones in function of their relative importance. One the main details is the detail $\mathrm{N}^{\circ} 20$ which is also selected for its simplicity.

\subsection{Detail $N^{\circ} 20$}

It is made of one central plate on which two lateral plates are welded. Moreover the real geometry of the weld were measured. Twenty four specimens with three different plate thicknesses were tested (Figure 5).

Three thickness sizes of specimens were used in the experiments: $6.35 \mathrm{~mm}, 12.7 \mathrm{~mm}$ and $25.4 \mathrm{~mm}$ for loading plates and $7.9 \mathrm{~mm}, 15.9 \mathrm{~mm}$ and $31.8 \mathrm{~mm}$ for corresponding centered plates.

Three different materials were always considered: base material (for detail No. 20, ASTM A-36 steel), weld metal (with Shielded Metal Arc Welding method), heat affected zone.

The experimental set up in the tension-compression machine, implies that the grips induce important initial stresses which were measured thanks to four strain gages located in the vicinity of the weld on the lateral plates.

Within all details, three materials were mechanically characterized: the base metal, the weld and the HAZ heat affected zone.

During each test, a fluctuating tensile load was applied: a mean stress level varying 0 and $144.5 \mathrm{MPa}$ with a random amplitude block (these stresses result from a long analysis on real naval structure). In principle, such a block was applied several times until one crack is initiated or the maximum number of 1500 times (which represents 125 years on the real structure). During the tests, special cares on the critical zones were taken, as these zones are the most sensitive to the crack initiation damage. For each sample, we have thus the geometry, the real loading, the place where cracks appear (but unhappily, the initial stresses due to the weld were not measured).

\subsection{Modelling and meshing with ALGOR/AUTOCAD}

Script files for the design system SD3 from Algor were written to allow the systematic input of the geometry and the mesh of the specimen according the given experimental data. It was taken into account that three materials had to be differentiated.

The Heat Affected Zone was not perfectly described in the report. It was guessed according the other geometrical information. The system Algor proposes several mesh generators. For a 2D structure, it uses Supergen (these quoted options and programs are no more available in the new integrated version).

In order to take into account the different materials and to have a thinner mesh around the sensitive points, it is necessary to give some more information (Figure 6). 
(a)

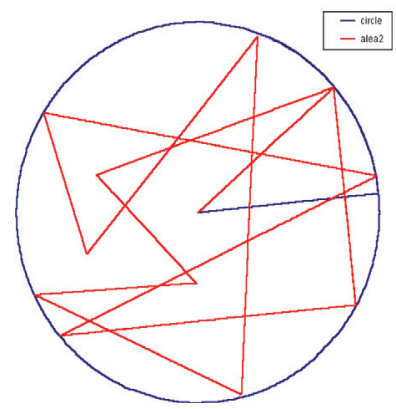

(b)

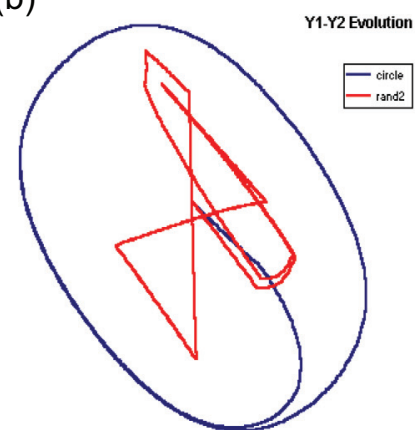

(c)

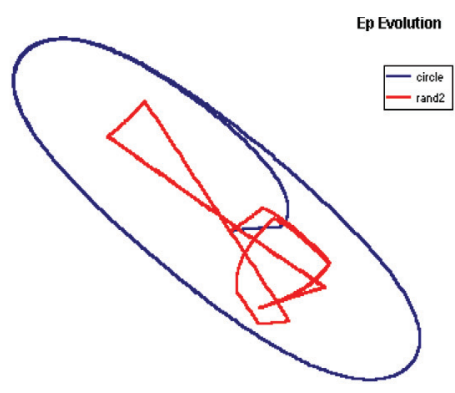

Figure 3. Any stress path is bounded by its boundary "circular" loading (blue: circle loading; red: random loading. (a) In the stress space, (b) in the transformed parameters space (c) in the plastic strain space.
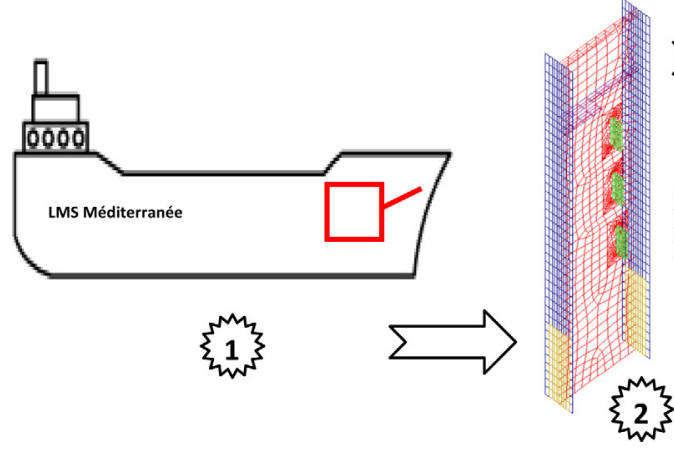

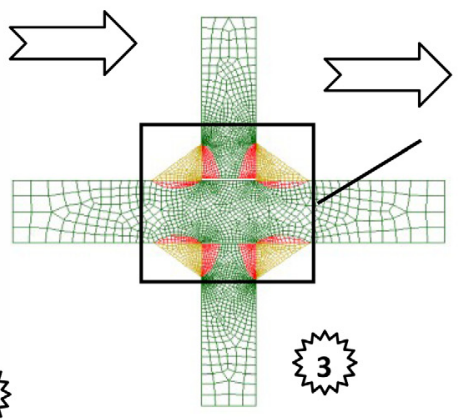

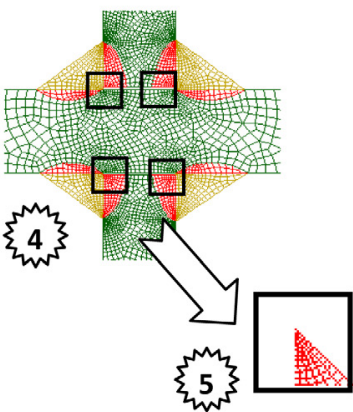

Figure 4. Multi-level analysis. (1) General structure, (2) 3D substructure, (3) 2D substructure, (4) window, (5) region.

\subsubsection{Elastic analysis with NISA from EMRC CRANES}

It is necessary to edit the NISA file within the preprocessor DISPLAY, to define the materials, the boundary conditions. It is also necessary to introduce at first the initial stresses due to the grips and the misalignment. As underlined in the report, these stresses may be important although the care brought by the technicians.

It is, at last, necessary to define the loading on the specimen. For elastic only some unit loadings are introduced and then combined. For the elastoplastic analysis, the full time history of the loading is introduced.

Without giving details, we obtained the results for the eight sensitive zones (IJP and TOE) and also for four other randomly chosen zones within the specimen. The size of the zones or windows has been taken as a fundamental parameter of the study, four sizes were retained ( $1 \mathrm{~mm}, 2 \mathrm{~mm}, 4 \mathrm{~mm}$ and $8 \mathrm{~mm}$ ).

The results of these analysis consist into the deduction of the initial stress field due to the grips and the applied stress field due to the random traction for each of the 12 regions and for each of the 3 differentiated materials.

\subsubsection{Elastoplastic analysis}

The same samples with the same meshes but with a special loading path were analyzed. Indeed we added to the initial stress field due to the grip, the particular real load history as described in the report [9] and we assumed that the stress field due to the welding (which is not known) was equal to zero.

As with all the samples, the elastic shakedown behavior was reached, we kept from the elastoplastic analysis, only the residual stress field which can then be treated as the initial stress field due to the grips during the elastic analysis.

The same intelligent descriptors were computed within the same windows around the same points in the specimens.

We also performed the incremental inelastic analysis during the random loading as described in the report and we considered the stress field at the end of the loading.

The execution times were very important with NISA. The simplified inelastic analysis, ZAC (Zarka-Casier) was at last performed. All details may be found in the book [1]. A copy of this book may be sent free on request to jose.zarka@gmail.com.

\subsubsection{Classical fatigue analysis}

From the stress field calculated all along the loading path, special tools such as ENDURE from EMRC and SOLSTICE from LMS allow classical numerical evaluation of the endurance limit of the part. Our objective is not to compare various criteria between them or to criticize them. We have taken the Dang-Van criterion because it is reliable and easy to use; but its value is weighted. It is applied to each meshing 

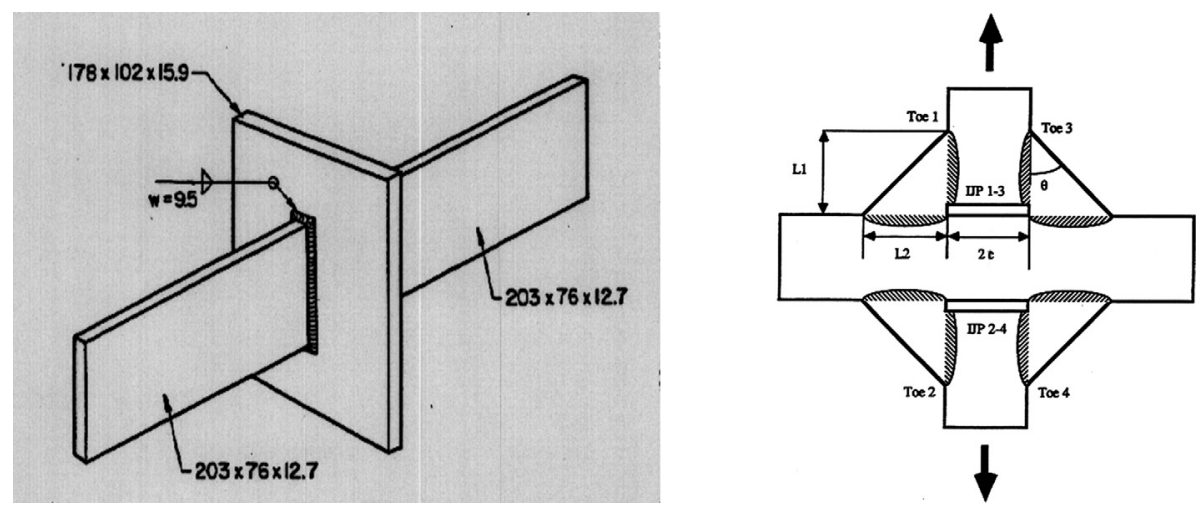

Figure 5. Detail n`20 with the critical points (from S.K. PARK).

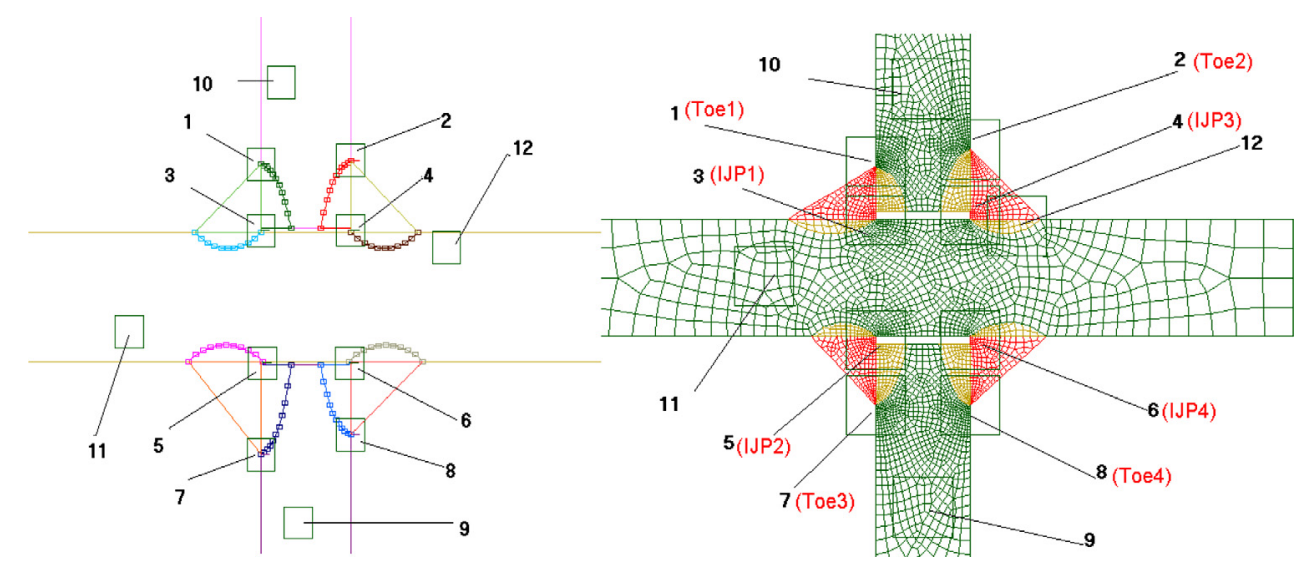

Figure 6. Meshes of the detail $\mathrm{N}^{\circ} 20$ in the various zones and position of the $2 \mathrm{D}$-windows.

node where the track of loading in diagram $\tau-p$ is drawn and the value of criterion $F^{\mathrm{DV}}$ is calculated. Critical points are either in $F^{\mathrm{DV}}>1$ or in $\max \left(F^{\mathrm{DV}}\right)$ if no point violates the criterion.

In ENDURE, we have decided to use a more classical stress-life type approach (2) which predicts the fatigue life of a part under cyclic loading.

\subsection{Definition of the intelligent descriptors}

As underlined all along this text, our objective being to be able to describe this detail 20 as any general structure. We have to make its analysis at three successive multi-levels. These analysis have to be made with corresponding mesh sizes and some submodelling option where the results on the boundary of the coarse mesh are applied to the more refined mesh.

For us, one case, will consist at the particular behavior of one material within one window.

At our actual stage of analysis of the detail $\mathrm{N}^{\circ} 20$, we have performed already levels 2 and 3; it is now necessary to describe in an intelligent way the results obtained in each window for each material:

(i) the material characteristics during cyclic loadings, cyclic curves at different strain rate, Wöhler curves and eventually also the rupture properties; (ii) the stress field with its evolution;

(iii) some other factors such as given by the experts in fatigue/rupture or deduced from general tools.

With such a description, the conclusions will be:

(i) Failure or Not failure,

(ii) if Failure, what is the fatigue life.

There are some natural methods to represent the material properties with some discrete parameters or descriptors. The most difficult part is the representation of the evolution of the stress field which are here the initial stresses due to the welding (unknown in this study), the initial stresses due to the grips, and the applied random tensile load.

\subsubsection{Material descriptors}

We used only these seven classical terms:

$\left(\sigma_{\mathrm{u}}\right)$ ultimate stress, $\left(\sigma_{y}^{\prime}\right)$ elastic cyclic limit, $(b)$ fatigue strength exponent, $(c)$ fatigue ductility exponent, $\left(n^{\prime}\right)$ cyclic hardening exponent, $\left(k^{\prime}\right)$ coefficient cyclic strength coefficient, $\left(\sigma_{\mathrm{f}}^{\prime}\right)$ fatigue strength coefficient.

\subsubsection{Stress field descriptors}

We suppose that the stress field at a given time is represented in any point by three scalar fields: its first invariant $I_{1}$, 
Table 1. The 27 min-max-moy descriptors to represent the evolution of the stress field within the region $\Omega$.

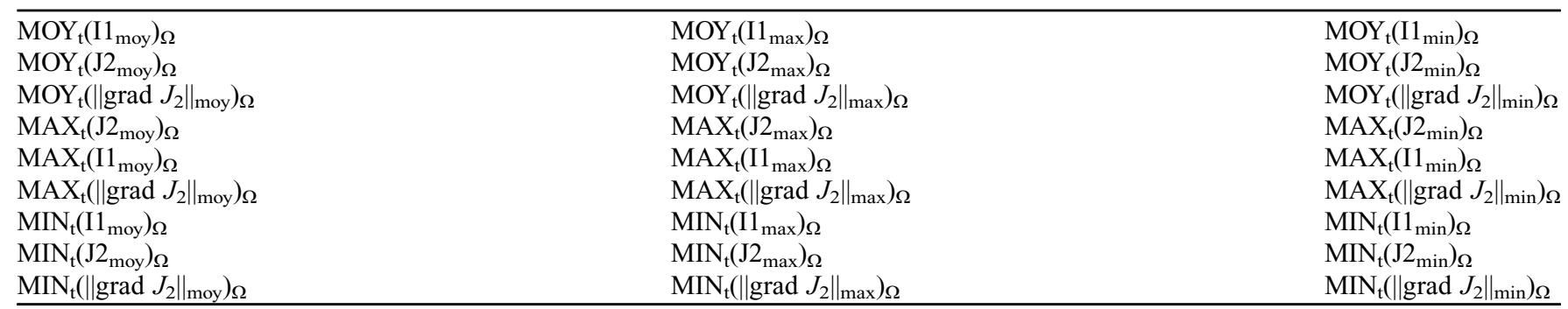

Table 2. The 45 surface descriptors to represent the evolution of the stress field within the region $\Omega$.

\begin{tabular}{|c|c|c|c|c|}
\hline $\operatorname{MOY}_{\mathrm{t}}\left(\mathrm{I1}_{\text {lay } 1}\right)_{\Omega}$ & $\operatorname{MOY}_{\mathrm{t}}\left(\mathrm{I}_{\text {lay } 2}\right)_{\Omega}$ & $\mathrm{MOY}_{\mathrm{t}}\left(\mathrm{I}_{\text {lay3 } 3}\right)_{\Omega}$ & $\operatorname{MOY}_{\mathrm{t}}\left(\mathrm{I}_{\text {lay } 4}\right)_{\Omega}$ & $\operatorname{MOY}_{t}\left(\mathrm{I}_{\text {lay } 5}\right)_{\Omega}$ \\
\hline $\operatorname{MOY}_{\mathrm{t}}\left(\mathrm{J} 2_{\text {lay } 1}\right)_{\Omega}$ & $\operatorname{MOY}_{t}\left(J 2_{\text {lay } 2}\right)_{\Omega}$ & $\operatorname{MOY}_{t}\left(J 2_{\text {lay } 3}\right)_{\Omega}$ & $\operatorname{MOY}_{t}\left(J 2_{\text {lay } 4}\right)_{\Omega}$ & $\operatorname{MOY}_{\mathrm{t}}\left(\mathrm{J} 2_{\text {lay } 5}\right)_{\Omega}$ \\
\hline $\operatorname{MAX}_{\mathrm{t}}\left(\mathrm{I}_{\text {lay } 1}\right)_{\Omega}$ & $\operatorname{MAX}_{\mathrm{t}}\left(\mathrm{I}_{\text {lay } 2}\right)_{\Omega}$ & $\operatorname{MAX}_{\mathrm{t}}\left(\mathrm{I}_{\text {lay } 3}\right)_{\Omega}$ & $\operatorname{MAX}_{\mathrm{t}}\left(\mathrm{I} 1_{\text {lay } 4}\right)_{\Omega}$ & $\operatorname{MAX}_{\mathrm{t}}\left(\mathrm{I} 1_{\text {lay } 5}\right)_{\Omega}$ \\
\hline $\operatorname{MAX}_{\mathrm{t}}\left(\left\|\operatorname{grad} J_{2}\right\|_{\text {lay } 1}\right)_{\Omega}$ & $\operatorname{MAX}_{\mathrm{t}}\left(\left\|\operatorname{grad} J_{2}\right\|_{\text {lay2 } 2}\right)_{\Omega}$ & $\operatorname{MAX}_{\mathrm{t}}\left(\left\|\operatorname{grad} J_{2}\right\|_{\text {lay } 3}\right)_{\Omega}$ & $\operatorname{MAX}_{\mathrm{t}}\left(\left\|\operatorname{grad} J_{2}\right\|_{\text {lay } 4}\right)_{\Omega}$ & $\operatorname{MAX}_{\mathrm{t}}\left(\left\|\operatorname{grad} J_{2}\right\|_{\text {lay } 5}\right)_{\Omega}$ \\
\hline $\operatorname{MIN}_{\mathrm{t}}\left(\mathrm{I} 1_{\text {lay } 1}\right)_{\Omega}$ & $\mathrm{MIN}_{\mathrm{t}}\left(\mathrm{I}_{\text {lay } 2}\right)_{\Omega}$ & $\mathrm{MIN}_{\mathrm{t}}\left(\mathrm{I}_{\text {lay } 3}\right)_{\Omega}$ & $\operatorname{MIN}_{\mathrm{t}}\left(\mathrm{I}_{1 \text { lay } 4}\right)_{\Omega}$ & $\mathrm{MIN}_{\mathrm{t}}\left(\mathrm{I} 1_{\text {lay } 5}\right)_{\Omega}$ \\
\hline $\operatorname{MIN}_{\mathrm{t}}\left(\mathrm{J} 2_{\text {lay } 1}\right)_{\Omega}$ & $\operatorname{MIN}_{\mathrm{t}}\left(\mathrm{J} 2_{\text {lay } 2}\right)_{\Omega}$ & $\operatorname{MIN}_{\mathrm{t}}\left(\mathrm{J} 2_{\text {lay } 3}\right)_{\Omega}$ & $\operatorname{MIN}_{\mathrm{t}}\left(\mathrm{J} 2_{\text {lay } 4}\right)_{\Omega}$ & $\operatorname{MIN}_{\mathrm{t}}\left(\mathrm{J} 2_{\text {lay5 } 5}\right)_{\Omega}$ \\
\hline
\end{tabular}

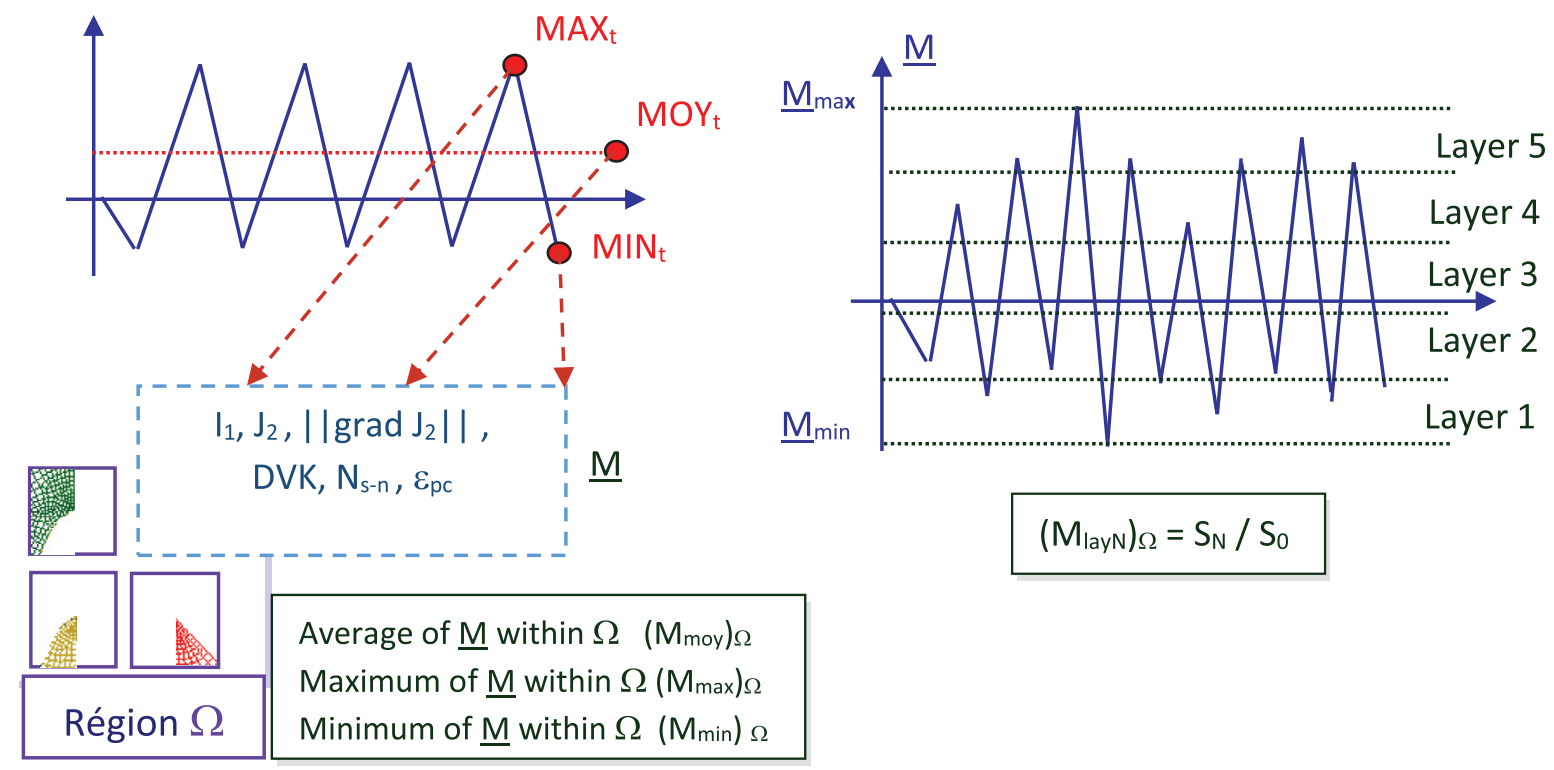

Figure 7. Synopsis of the definition of the intelligent descriptors.

the second invariant of its deviatoric part $J_{2}$ and the norm of the gradient of $J_{2}$

$$
\left\|\operatorname{grad} J_{2}\right\|=\sqrt{\left(\frac{\partial J_{2}(x, y)}{\partial x}\right)^{2}+\left(\frac{\partial J_{2}(x, y)}{\partial y}\right)^{2}}
$$

This choice is justified by the analysis of the fatigue criterion. Furthermore, it has the advantage of leading to reasonable calculation time since it is not necessary to search critical plan.

Also, it is necessary to describe the evolution with time of the fields associated to the stress field.

Although it was indicated in the Illinois report that the tensile load was a random load during their tests, we choose to represent the evolution of the stress field for three positions of an equivalent radial cyclic loading thanks to our equivalence rule at the structure level $[\mathrm{xx}]$ :

(i) at the average stress of applied loading ( $\mathrm{MOY}_{\mathrm{t}}$ ),

(ii) at maximum of applied loading $\left(\mathrm{MAX}_{\mathrm{t}}\right)$,

(iii) at minimum of applied loading $\left(\mathrm{MIN}_{\mathrm{t}}\right)$.

Our purpose remains the description of a general case of fatigue analysis inside a zone $\Omega$ defined for a given material within a $2 D$ window with a give size.

The local solution of the stress field highly depends on the finite element mesh. We suppose the mesh is made correctly around the critical zones. A good representation of properties 
associated to the stress field should allow taking into account or limiting the errors due to mesh. Here a representation quite simple is used to characterize at any moment a scalar field $M$ with an unknown error [10]: we think that it is acceptable (we don't know however the real level of error).

According to Hablot [10], if $M$ is one of the three scalar fields $I_{1}, J_{2}$ or $\left\|\operatorname{grad} J_{2}\right\|$, its description at any time $\left(\mathrm{MOY}_{\mathrm{t}}\right.$, $\mathrm{MAX}_{\mathrm{t}}, \mathrm{MIN}_{\mathrm{t}}$ ) in the region $\Omega$ is first made by introduction of three quantities [8]:

- the average value of $M$ in $\Omega$ : $\left(M_{\text {moy }}\right)_{\Omega}$,

- the maximum value of $M$ in $\Omega:\left(M_{\max }\right)_{\Omega}$,

- the minimum value of $M$ in $\Omega:\left(M_{\min }\right)_{\Omega}$.

We use other descriptors to characterize the scalar field $M(\underline{x})$ varying between $M_{\min }$ and $M_{\max }$. They are surface descriptors which will give indications on the distribution of the field $M(\underline{x})$ in the region $\Omega$. They are noted $M_{\text {lay } 1}$, $M_{\text {lay2}}, \ldots, M_{\text {lay } n}$ and are defined according to following method:

(i) the interval $\left[M_{\min } ; M_{\max }\right]$ is divided in $n$ equal segments $\left[M_{\min } ; M_{i+1}\right]$,

(ii) the relative surfaces $\left(S_{i} / S_{0}\right)$ in the window $\Omega$ for which the scalar $M$ is included in the $i$ th interval $\left[M_{i} ; M_{i+1}\right]$ is calculated, where $S_{0}$ is the surface of the concerned region.

In this study we have selected five intervals $(n=5)$. These quantities can be considered as elementary statistics of the field $M(\underline{x})$ in the region $\Omega$. In total, 72 descriptors (Tables 1 and 2) are so introduced to represent the evolution of the stress field within the region $\Omega$ [8].

\subsubsection{Fatigue descriptors}

We keep three fatigue characteristics to describe [8]:

- The fatigue function $F^{\mathrm{DV}}$ (calculated only for Dang Van criterion [8]);

- The number of cycles at the crack initiation $(N$, for stress-life approach);

- and the norm of loading block $\left(\varepsilon_{\mathrm{pc}}\right.$ ou $\left.W_{\mathrm{D}}\right)$.

These three characteristics are also represented by their average, maximal and minimal values in region $\Omega$ (Figure 7 ).

So nine new descriptors are introduced:

- Three descriptors for the fatigue function $F^{\mathrm{DV}}$ :

$$
\left(F_{\mathrm{m} 0 \mathrm{y}}^{\mathrm{DV}}\right)_{\Omega},\left(F_{\min }^{\mathrm{DV}}\right)_{\Omega},\left(F_{\max }^{\mathrm{DV}}\right)_{\Omega}
$$

- Three descriptors for the fatigue life given by the stresslife approach:

$$
\left(N_{\mathrm{m} 0 \mathrm{y}}^{S-N}\right)_{\Omega},\left(N_{\min }^{S-N}\right)_{\Omega},\left(N_{\max }^{S-N}\right)_{\Omega}
$$

Table 3. Comparison between the reliabilities of the various

\begin{tabular}{|c|c|c|}
\hline File Des_Ela1Pro.out & $\mathrm{C} 1$ & $\mathrm{C} 2$ \\
\hline True positive proportion & 0.8381 & 0.9348 \\
\hline False positive proportion & 0.0652 & 0.1619 \\
\hline File Des_Ela2Pro. out & $\mathrm{C} 1$ & $\mathrm{C} 2$ \\
\hline True positive proportion & 0.8404 & 0.9796 \\
\hline False positive proportion & 0.0204 & 0.1596 \\
\hline File Des_Ela4Pro out & $\mathrm{C} 1$ & $\mathrm{C} 2$ \\
\hline True positive proportion & 0.8003 & 0.98 \\
\hline False positive proportion & 0.02 & 0.1997 \\
\hline File Des_Ela8Pro. out & $C 1$ & $C 2$ \\
\hline True positive proportion & 0.8408 & 0.98 \\
\hline False positive proportion & 0.02 & 0.1592 \\
\hline File Des_Ela10Pro. out & $\mathrm{C} 1$ & $\mathrm{C} 2$ \\
\hline True positive proportion & 0.7392 & 0.94 \\
\hline False positive proportion & 0.06 & 0.2608 \\
\hline File Desc_cyc1pro. out & $\mathrm{C} 1$ & $\mathrm{C} 2$ \\
\hline True positive proportion & 0.877 & 0.9111 \\
\hline False positive proportion & 0.0889 & 0.123 \\
\hline File Desc_cyc2pro. out & $\mathrm{C} 1$ & $\mathrm{C} 2$ \\
\hline True positive proportion & 0.871 & 0.9388 \\
\hline False positive proportion & 0.0612 & 0.129 \\
\hline File Desc_cyc4pro. out & $\mathrm{C} 1$ & $\mathrm{C} 2$ \\
\hline True positive proportion & 0.8771 & 0.94 \\
\hline False positive proportion & 0.06 & 0.1229 \\
\hline File Desc_Cyc8pro. out & C1 & $\mathrm{C} 2$ \\
\hline True positive proportion & 0.8035 & 0.98 \\
\hline False positive proportion & 0.02 & 0.1965 \\
\hline File nDes_Ran10pro. out & $\mathrm{C} 1$ & $\mathrm{C} 2$ \\
\hline True positive proportion & 0.738 & 0.9167 \\
\hline False positive proportion & 0.0833 & 0.262 \\
\hline File nDesc_cyc10pro. out & $\mathrm{C} 1$ & $\mathrm{C} 2$ \\
\hline True positive proportion & 0.8402 & 0.92 \\
\hline False positive proportion & 0.08 & 0.1598 \\
\hline File Desc_zac1pro. out & $\mathrm{C} 1$ & $\mathrm{C} 2$ \\
\hline True positive proportion & 0.873 & 0.913 \\
\hline False positive proportion & 0.087 & 0.127 \\
\hline File Desc_zac2pro. out & $\mathrm{C} 1$ & $\mathrm{C} 2$ \\
\hline True positive proportion & 0.8333 & 0.9388 \\
\hline False positive proportion & 0.0612 & 0.1667 \\
\hline File Desc_zac4pro. out & $\mathrm{C} 1$ & $\mathrm{C} 2$ \\
\hline True positive proportion & 0.8312 & 0.98 \\
\hline False positive proportion & 0.02 & 0.1688 \\
\hline File Desc_zac8pro. out & $\mathrm{C} 1$ & $\mathrm{C} 2$ \\
\hline True positive proportion & 0.8177 & 0.92 \\
\hline False positive proportion & 0.08 & 0.1823 \\
\hline File Desc_zac10pro. out & $\mathrm{C} 1$ & $\mathrm{C} 2$ \\
\hline True positive proportion & 0.8333 & 0.9388 \\
\hline False positive proportion & 0.0612 & 0.1667 \\
\hline File nDes_Ran1pro. out & $\mathrm{C} 1$ & $\mathrm{C} 2$ \\
\hline True positive proportion & 0.928 & 0.9333 \\
\hline False positive proportion & 0.0667 & 0.072 \\
\hline File nDes_Ran2pro. out & $\mathrm{C} 1$ & $\mathrm{C} 2$ \\
\hline True positive proportion & 0.8737 & 0.8776 \\
\hline False positive proportion & 0.1224 & 0.1263 \\
\hline File nDes_Ran4pro. out & $\mathrm{C} 1$ & $\mathrm{C} 2$ \\
\hline True positive proportion & 0.8886 & 0.84 \\
\hline False positive proportion & 0.16 & 0.1114 \\
\hline File nDes_Ran8pro. out & $\mathrm{C} 1$ & $\mathrm{C} 2$ \\
\hline True positive proportion & 0.8359 & 0.96 \\
\hline False positive proportion & 0.04 & 0.1641 \\
\hline
\end{tabular}
learnings.

File Des_Ela1Pro.out

True positive proportion

eroportion

True positive proportion

False positive proportion

File Des ElasPro. out

True positive proportion

False positive proportion

True positive proportion

False positive proportion

True positive proportion

False positive proportion

File Desc_Cyc8pro. out

True positive proportion

False positive proportion

File Desc_zac1pro. out

True positive proportio

positive proportio

False positive proportion

File Desc zac4pro. out

True positive proportion

False positive proportio

False posi

File nDes_Ranlpro. out

True positive proportion

False positive proportio

File nDes_Ran4pro. out

True positive proportion

False positive proportion

True positive proportion

False positive proportion 
- Three descriptors for the norm of loading block:

$$
\left(\varepsilon_{\mathrm{pc}_{\mathrm{m} 0 \mathrm{y}}}\right)_{\Omega},\left(\varepsilon_{\mathrm{pc}_{\min }}\right)_{\Omega},\left(\varepsilon_{\mathrm{pc}_{\max }}\right)_{\Omega}
$$

\subsection{Data base and generation of the rules}

\subsubsection{Compilation of data and automatic learning}

Several details were tested [8]. For each size of the window, several text files are induced. All these text files are merged. The observed conclusions are added. The data base has been so created. In the Excel files name1.xls, name2.xls, name4.xls and name8.xls, name10.xls the results of all the analysis corresponding to a window size of $1 \mathrm{~mm}, 2 \mathrm{~mm}, 4 \mathrm{~mm}$, $8 \mathrm{~mm}$ and $10 \mathrm{~mm}$ are given.

We recall that in our approach, each subregion i.e. one material in one region, is considered as an independent case/example for which we have its own intelligent descriptors and its two conclusions Failure or No failure and when there is failure, the number of cycles or number of blocks.

We used LES from the Ecole Polytechnique and Neuroshell from Wards systems to extract automatically the rules [8]. Only the learning of Failure or No Failure has been done for the moment.

We also used the Probabilistic Neural Networks (PNN) which are able to train on sparse data sets and to separate data into a specified number of output categories (Table 3).

\section{Conclusions}

From these results, the best window size has to be taken equal to $8 \mathrm{~mm}$ during the elastic analysis. Moreover, the simplified and elastoplastic analysis give almost the same reliability than the elastic one. Then only elastic analysis for any new structure with a window size of $8 \mathrm{~mm}$ have to be taken.

The rules which were generated on this detail, can now be used to analyze any other structure.
However, more experimental tests are still necessary to qualify fully our approach.

A software, FATPRO, based on this work has been developed at MZ Intelligent Systems, the brochure in [2].

Acknowledgements. This paper was written in part during the regular visits of the first author to UCSD's Center of Excellence for Advanced Materials, under ONR contract N00014-96-1-0631 (R. Barsoum, Coordinator) to the University of California San Diego and for the other part by the second author during his $\mathrm{PhD}$ thesis under the sponsorship of Ligeron S.A. (A. Azarian, Coordinator). We want to thank Prof S. Nemat-Nasser for his helpful discussions.

\section{References}

1. Zarka J, et al. 1990. A new approach in inelastic analysis of structures. France: CADLM.

2. MZ Intelligent Systems, "FatPro" Software. Available at http:// www.mzintsys.com/our_products_fatpro.html.

3. Zarka J, Navidi P. 2000. Intelligent optimal design of materials and structures. MZ Intelligent Systems: France, http://www.mzintsys.com/publication/Intelligent_Optimimum_ Design_J-Zarka_\&_P-Navidi.pdf.

4. Glinka G, KAM JCP. 1987. Rainflow counting algorithm for very long stress histories, International Journal of Fatigue, 9, 223-228.

5. Lemaitre J, Chaboche J-L. 1988. Mécanique des matériaux solides. France: Dunod.

6. Lalanne C. 1999. Dommage par fatigue - Vibrations et chocs mécaniques. Hermès: Paris.

7. Dang-Van K. 1973. Sciences et Techniques de l'Armement. France. p. 647-722.

8. Karaouni H. Phd thesis, Ecole Polytechnique X, France, 2001.

9. Park SK, Lawrence FV, Fatigue characterisation of fabricated ship details for design - Phase 2, 1988.

10. Hablot J-M. 1990. Phd thesis, ENPC, France. 